\title{
Nemaline rod myopathy treated with L-tyrosine to relieve symptoms in a neonate
}

\author{
Suzan Sahin M.D. ${ }^{a}$, Mehmet Y. Oncel M.D. ${ }^{a}$, Duygu Bidev M.D. ${ }^{a}$, Nilufer Okur M.D. ${ }^{a}$, Beril Talim M.D. ${ }^{b}$ and \\ Serife S. Oguz M.D. ${ }^{a}$
}

\begin{abstract}
Nemaline myopathy (NM) is a heterogeneous disorder defined by the presence of rod-shaped structures known as nemaline bodies or rods. The diagnosis is based on muscle weakness, combined with visualization of nemaline bodies on muscle biopsy. There is no curative treatment for nemaline myopathy. Therapeutic strategies for this condition are symptomatic and empirical. Herein, we present a newborn with severe respiratory failure and generalized muscle weakness, who was diagnosed as NM by muscle biopsy. The patient experienced remarkable decrease in sialorrhea and improvement of spontaneous movements after L-tyrosine treatment.

This case is presented to emphasize the importance of muscle biopsy in the differential diagnosis of severe hypotonia during neonatal period and a possible benefit of L-tyrosine supplementation for decreasing sialorrhea and restoring muscle strength.

Key words: Nemaline myopathy; muscle hypotonic; infant, newborn; L-tyrosine.
\end{abstract}

http:/ / dx.doi.org/10.5546/ aap.2019.eng.e382

To cite: Sahin S, Oncel MY. Bidev D, Okur N, et al. Nemaline rod myopathy treated with L-tyrosine to relieve symptoms in a neonate. Arch Argent Pediatr 2019;117(4):e382-e386.

\section{INTRODUCTION}

Nemaline myopathy (NM) is a clinically and genetically heterogeneous disorder defined by the presence of rod-shaped structures known as nemaline bodies or rods, on muscle biopsy. The diagnosis is based on muscle weakness, combined with visualization of nemaline bodies on muscle biopsy. In addition to generalized weakness,

a Department of Neonatology, Zekai Tahir Burak Maternity Teaching Hospital, Ankara; Turkey.

b. Department of Pediatrics, Pathology Unit, Hacettepe University Faculty of Medicine, Ankara; Turkey.

E-mail address:

Suzan Sahin, M.D.: suzan_balkan@yahoo.com

Funding: None.

Conflict of interest: None.

Received: 4-24-2018

Accepted: 1-16-2019 bulbar dysfunction causes swallowing difficulties and drooling, and may predispose to aspiration of oral and pharyngeal secretions. ${ }^{1}$

All molecularly characterized forms of nemaline myopathy are autosomal, but inheritance can be recessive or dominant. The prevalence of each form is difficult to determine, as many cases are not molecularly resolved. ${ }^{2,3}$

Nemaline myopathy most commonly has a congenital onset, but varies clinically from fetal akinesia sequence to mild childhood-onset forms, and even to adult-onset cases, most of which, as stated, may not be genetic in origin. Electromyography (EMG) is nonspecific. Muscle imaging (muscle ultrasound and MRI) can help to differentiate between different forms of congenital myopathy. Selective muscle involvement on MRI can be suggestive of a specific disease genehowever the specificity is variable and imaging is usually interpreted in conjunction with clinical phenotype and results of muscle biopsy to prioritize gene testing. ${ }^{4}$ Muscle biopsy is the mainstay of reaching the diagnosis of a specific form of congenital myopathy. ${ }^{5}$ In nemaline myopathy, muscle biopsy specimens show numerous thread like inclusions on modified Gomori trichrome staining. ${ }^{6}$

Patient care is based on managing the clinical symptoms. ${ }^{2}$ L-tyrosine may prevent aspiration by reducing pharyngeal secretions and drooling. Improvement in muscle strength is also reported in several cases. ${ }^{7,8}$

We present a newborn with severe respiratory failure and generalized muscle weakness, who was diagnosed as NM by muscle biopsy. The patient experienced remarkable decrease in drooling and improvement of spontaneous movements after L-tyrosine treatment.

\section{CASE}

A baby girl, weighted 2000 grams, born at the $33^{\text {rd }}$ gestational week by cesarean section as the first child of the family, was hospitalized due to severe neonatal hypotonia and respiratory failure. Apgar scores were 3 and 6 at minute 1 and 5, respectively. The history of the mother, 
who was 17 years old, revealed only decreased sensation of fetal movements during the last trimester of the pregnancy. There was seconddegree consanguinity between the parents and there was no family history of a similarly affected baby or neuromuscular disease.

Physical examination revealed severe hypotonia, which was prominent in facial muscles with prominently distal weakness and a frog-leg posture, peculiar hands with lack of clenching of the index finger on both sides, hyporeflexia and decrease respiratory effort (Figure 1 and 2). No dysmorphic features was observed. Sensory evaluation was normal. Echocardiography showed minimal tricuspid insufficiency and mild dynamic obstructive gradient in the left ventricular outlet, which resolved during the follow-up. On cranial ultrasonography, a grade 1 periventricular hemorrhage was observed. As for differential diagnosis, no positivity in IgM levels was observed with the TORCH serology. Regarding congenital muscular dystrophies, muscle enzymes were within the normal limits. Possible metabolic disorders, which may present with infantile hypotonia, were excluded by detailed metabolic screening tests and neither acidosis nor hyperlactic acidemia was detected. The karyotype analysis was $46, \mathrm{XX}$.

Cranial magnetic resonance imaging was consistent with the age of the baby and did not show any structural abnormality or finding suggestive for hypoxic ischemic encephalopathy.
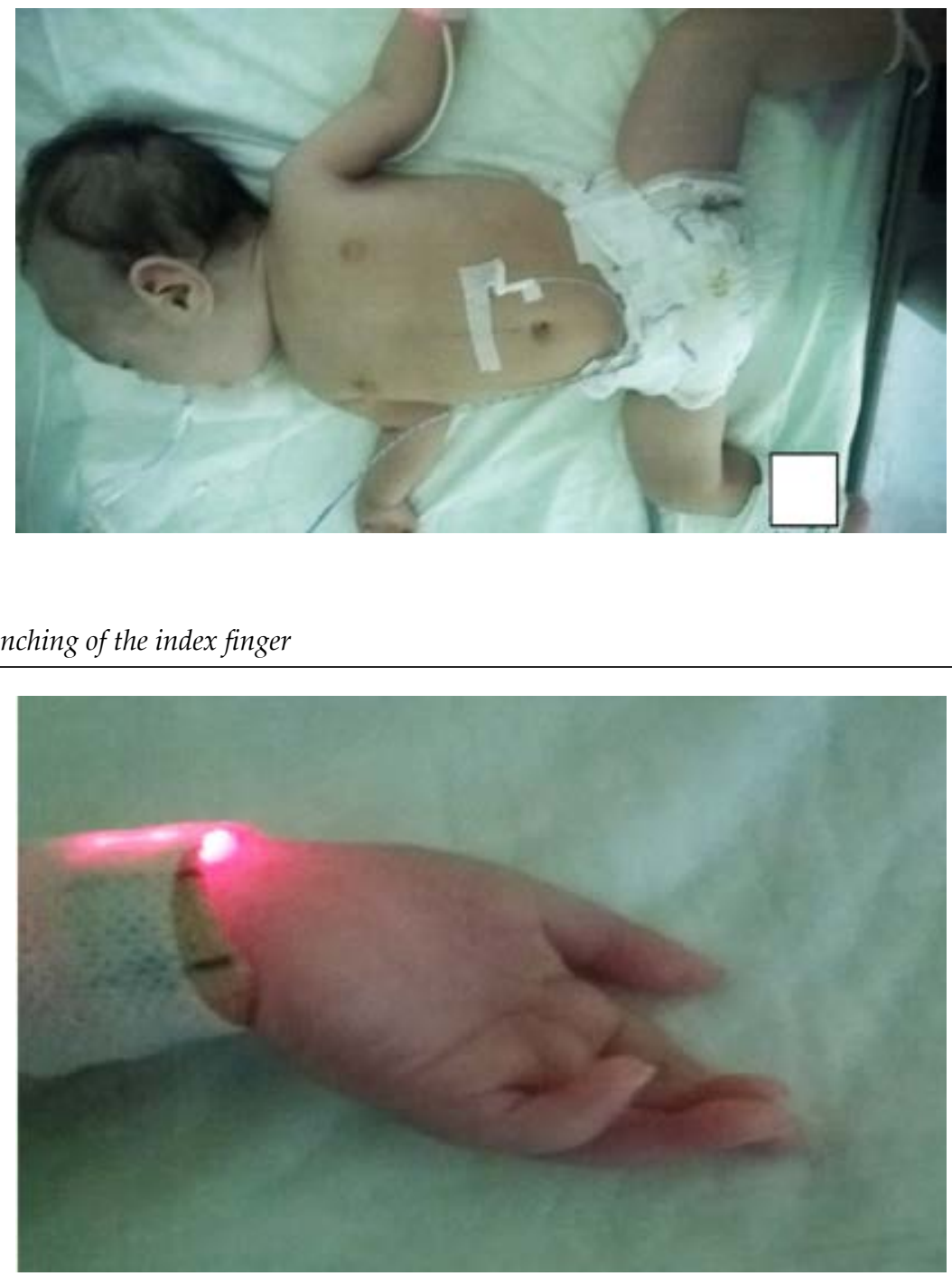
Denervation disorders and congenital myasthenic syndromes were included in the differential diagnostic work-up. Nerve conduction studies and specific investigations for these disorders could not be performed due to technical reasons. However, as we did not observe any tongue fasciculations and the degree of weakness of the baby was not increasing in correlation with active muscle movements, these group of disorders were excluded clinically. Genetic analysis for spinal muscular atrophy was negative. On the $65^{\text {th }}$ day of life, muscle biopsy was performed from the quadriceps muscle. There was prominent variation in fiber size and nemaline rods were detected mainly in atrophic fibers by modified Gomori trichrome staining (Figure 3). As the nebulin gene gives frames with distal weakness and it is of autosomal recessive (AR) inheritance, NEB-gene mutation was the most possible etiology but due to economic reasons, the parents did not accept genetic diagnosis.

Following the definitive diagnosis of nemaline myopathy by muscle biopsy, we started L-tyrosine treatment, which caused partial decrease in drooling and increase in the observed spontaneous extremity movements. Genetic counselling was given to the parents. The baby died on the $173^{\text {rd }}$ day of life due to sepsis.

\section{DISCUSSION}

Nemaline myopathy is a heterogeneous form of congenital myopathy characterized by a variable spectrum of clinical features, predominated in the severe form by profound muscle hypotonia and weakness accompanied by respiratory insufficiency. ${ }^{9}$ Bulbar dysfunction in NM causes swallowing difficulties and drooling, and may predispose to aspiration of oral secretions. To date, there are no curative treatments for NM. Therapeutic strategies for this condition are symptomatic and empirical. ${ }^{7}$

Most of the disorders which affect nervous or skeletal system, present with hypotonia, regardless of the location of primary abnormality. Besides, hypotonia may also be the symptom of most of the multisystem disorders of a newborn baby. The differential diagnosis between a primary central nervous system disorder and a neonatal muscle disorder requires a methodological approach including a detailed family, obstetric and delivery history and a careful examination. ${ }^{8}$ Muscle biopsy is usually left as the last step of diagnostic tests, in the differential diagnosis of infantile hypotonicity. In the presented case, we could confirm the diagnosis by an eventual muscle biopsy after a thorough investigation of all the possible disorders with a stepwise approach. The biopsy specimen of our patient showed the nemaline bodies (rods) as dark blue/purple structures scattered throughout the muscle fibers, mostly atrophic ones. Diagnosis may be difficult by light microscopy in the presence of rare fibers showing very few rods, especially in neonatal biopsies or in the presence of very atrophic fibers, as in our case. Nemaline rods must be

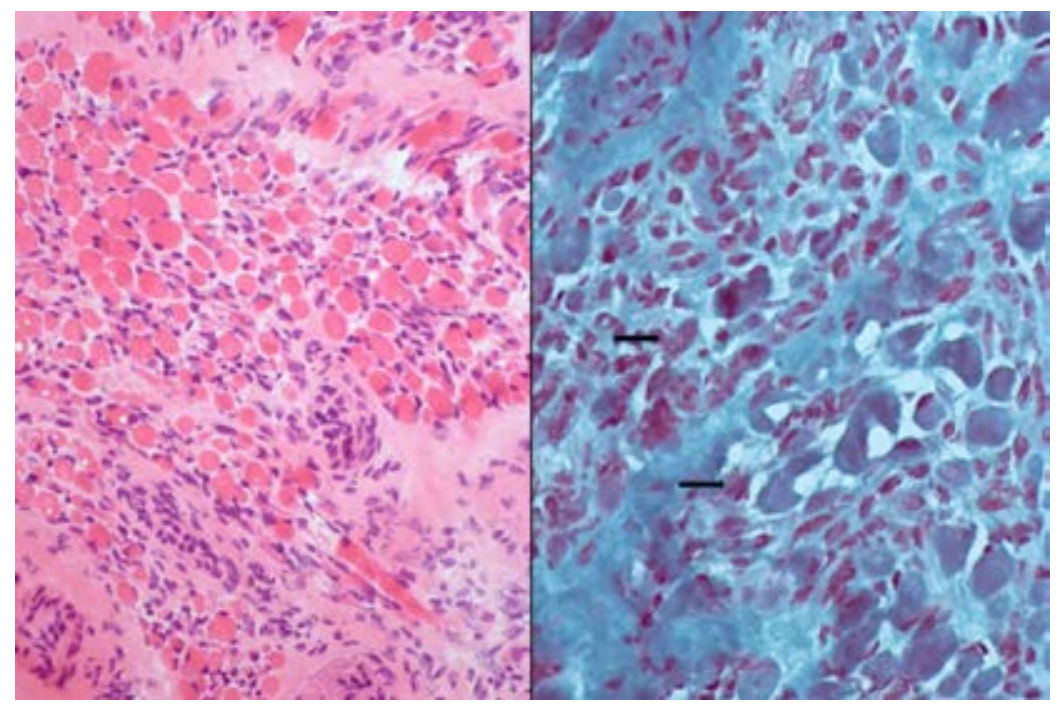


differentiated from mitochondria and cytoplasmic bodies. Electron microscopy also helps diagnosis but it was not available in our case. Nemaline rods are not specific to nemaline myopathy, can be seen in normal myotendinous junction, extraocular muscles and aging muscle and as a minor occurrence in several other myopathies. ${ }^{10}$ Infantile onset myopathy was excluded as our patient did not have dilated cardiomyopathy or arthrogryposis, adult onset myopathy was excluded according to the age group. For, HIV rod myopathy, the mother was negative for HIV serology.

Even if 10 genes have been found to cause nemaline myopathy, mutations in the NEB gene have been identified as a cause of about $50 \%$ of nemaline myopathy. Mutations in this gene can cause any form of the disorder, but most individuals with a NEB mutation have the typical congenital form. We aimed to support our clinical and pathological diagnosis by genetic diagnosis but as discussed earlier, it could not be possible in our case. ${ }^{11}$

Anticholinergic agents and salivary gland botulinum toxin injections are usually ineffective to decrease oral and pharyngeal secretions. ${ }^{12}$ But even if not certainly contraindicated, there are papers reporting severe and prolonged dysphagia due to local botulinum toxin injection to the cases with general weakness. Besides, the main cause of drooling in these patients is, not really hypersalivation, but poor control of oral secretions and insufficiency of muscle strength. Low amount of saliva has also its possible bad consequences in these patients. ${ }^{13}$ On the other hand, there are few reports regarding the possible positive effect of L-tyrosine on decreasing secretions and increasing muscle strength. ${ }^{7,8,14}$

Tyrosine is an aromatic amino acid derived in mammals from hydrolysis of dietary or tissue proteins or from hydroxylation of dietary or tissue phenylalanine. ${ }^{15}$ But tyrosine is also the starting point of the synthetic pathways producing catecholamines, thyroid hormone, and the melanin pigments. ${ }^{14}$ But it is not yet clear what the mechanism of action to achieve improvement is.

We observed partial decrease in the oral and pharyngeal secretions and increase in the observed spontaneous extremity movements after the initiation of L-tyrosine. Our experience provides support to previous evidence that L-tyrosine can improve bulbar and muscular function and increase cathecholamine-mediated sympathetic activity in the salivary glands, in patients with NM. On the other hand, in any case it should be clear that severe structural myopathies with respiratory compromise after a critical stage could also improve spontaneously. Due to our clinical diagnosis together with the results of the pathological examination, the change in the degree of hypotonia and the amount of drooling was attributed to the potential effect of L-tyrosine rather than to the spontaneous improvement of these clinical signs over time, which is rare to occur in severe congenital nemaline myopathy. ${ }^{11}$

L-tyrosine was safe and potentially effective treatment in this case but its use in similar cases needs further investigation.

\section{REFERENCES}

1. Ryan MM, Schnell C, Strickland CD, Shield LK, et al. Nemaline myopathy: a clinical study of 143 cases. Ann Neurol. 2001; 50(3):312-20.

2. Wallgren-Pettersson C, Sewry CA, Nowak KJ, Laing NG. Nemaline myopathies. Semin Pediatr Neurol. 2011; 18(4):230-8.

3. Jungbluth H, Sewry CA, Muntoni F. What's new in neuromuscular disorders? The congenital myopathies. Eur J Paediatr Neurol. 2003; 7(1):23-30.

4. North KN, Wang CH, Clarke N, Jungbluth H, et al. Approach to the diagnosis of congenital myopathies. Neuromuscul Disord. 2014; 24(2):97-116.

5. Dubowitz V, Sewry CA. Congenital myopathies. In Dubowitz V, Sewry CA. Muscle biopsy: a practical approach. 3rd ed. London: Saunders-Elservier; 2007.

6. SharmaMC, Jain D, Sarkar C, Goebel H. Congenital myopathies-a comprehensive update of recent advancements. Acta Neurol Scand. 2009; 119(5):281-92.

7. Ryan MM, Sy C, Rudge S, Ellaway C, et al. Dietary L-tyrosine supplementation in nemaline myopathy. J Child Neurol. 2008; 23(6):609-13.

8. Olukman O, Calkavur S, Diniz G, Unalp A, Atlihan F. Nemaline myopathy in a newborn infant: a rare muscle disorder. Neurol Neurochir Pol. 2013; 47(5):493-8.

9. Bojdo A, Obersztyn E, Wallgren-Pettersson C, Lehtokari $\mathrm{V}$, et al. Nemaline myopathy as a cause of neonatal hypotonia - with emphasis on personal experiences. Report of a family with two brothers affected. Med Wieku Rozwoj. 2009; 13(1):5-10.

10. Sewry CA, Wallgren-Pettersson C. Myopathology in congenital myopathies. Neuropathol Appl Neurobiol. 2017; 43(1):5-23.

11. Sanoudou D, Beggs AH. Clinical and genetic heterogeneity in nemaline myopathy-a disease of skeletal muscle thin filaments. Trends Mol Med. 2001; 7(8):362-8.

12. Brei TJ. Management of drooling. Semin Pediatr Neurol. 2003; 10(4):265-70.

13. Morgan AT, Dodrill P, Ward EC. Interventions for oropharyngeal dysphagia in children with neurological impairment. Cochrane Database Syst Rev. 2012; 10:CD009456.

14. Kalita D. A new treatment for congenital nonprogressive nemaline myopathy. J Orthomol Med. 1989; 4(2):70-4.

15. Scriver CR, Beaudet AL, Sly WS, VAlle D, et al. The Metabolic and Molecular Bases of Inherited Disease. 8th ed. New York: McGraw-Hill; 2001. 